\title{
Transverse Myelitis Associated with Mycoplasma Pneumoniae: Case Report
}

\author{
L. Heller, $M D^{1,2}$ O. Keren, $M{ }^{1,2}$ L. Mendelson, $M D,{ }^{1,2}$ G. Davidoff, $M D,{ }^{3,4}$ \\ ${ }^{1}$ Loewenstein Rehabilitation Hospital, Raanana, ${ }^{2}$ Sackler School of Medicine, Tel \\ Aviv University, Ramat-Aviv, Israel, ${ }^{3}$ Rehabilitation Medicine Service, Veterans \\ Administration Medical Center, ${ }^{4}$ Department of Physical Medicine and Rehabilitation \\ University of Michigan Medical Center Ann Arbor, Michigan, USA.
}

\section{Summary}

Acute transverse myelitis (ATM) is a disorder of the spinal region of the central nervous system. In the present case, the clinical course showed ATM associated with mycoplasma pneumoniae (MP) and little recovery; the girl was left with a flaccid paraplegia, and thus differed from other cases in the literature which all reported complete or nearcomplete recovery.

Key words: Acute transverse myelitis; Mycoplasma pneumoniae; Paraplegia.

Acute transverse myelitis (ATM) is a disorder of the spinal region of the central nervous system. Its clinical characteristics are bilateral motor and sensory lesions at a specific spinal level, and dysfunction of the rectal and urinary sphincters. This definition of ATM excludes those patients who have other known neurological disorders or who have shown evidence of an intra- or extra-medullary lesion (Tyler et al., 1986). The incidence of ATM is less than 2 cases per million (Berman et al., 1981) population per year. ATM is associated with many infections, mostly viral, and with vascular diseases, multiple sclerosis and malignancies (Lipton and Teasdall, 1973; Merli and Staas, 1985; Jain et al., 1983). The incidence of infectious disease preceding ATM is high and even higher in young people. Among patients affected by ATM, about one third fully recover, one third partially recover, while the rest remain permanently disabled or die (Berman et al., 1981).

Several clinical reviews and case reports have shown mycoplasma pneumonia (MP) to be a factor involved in various pathologies of the nervous system (Clyde, 1980; Behan et al., 1986). There is circumstantial evidence for a connection between mycoplasmal infection and ATM (Behan et al., 1986; McFarlane and Miller, 1984; Nicholson, 1977; Klimek et al., 1976). In the case to be described here, evidence is presented of antibodies associated with mycoplasma in the cerebrospinal fluid of a patient with ATM. The case is exceptional in that the 
patient failed to improve and has been left with a complete paraplegia without substantial recovery.

\section{Report of case}

A previously healthy, 13-year-old daughter of non-consanguinous parents was admitted to the medical centre complaining of weakness and paraesthesia in both legs. Two weeks earlier, she had fever, headaches and coughing with sputum production, which subsided after treatment with Moxypen (amoxycillin), $500 \mathrm{mg}$ po tid for 5 days. Three days prior to admission she had headaches, and 48 hours before admission complained of pain and numbness in both hips. She denied any episodes of fever, chills or diaphoresis. On admission to the emergency ward, the patient was found to have decreased air entry in the right hemi-thorax. Neurological examination allowed the patient to be fully conscious, and oriented with a grossly normal mental status. The cranial nerves, cerebellum and upper limb functions were normal. There was complete flaccid paraplegia with arreflexia and with urinary and fecal incontinence. The abdominal reflexes were absent and there was loss of sensation to light touch and pain from T6 caudally. She also had bladder and rectal incontinence. There was no evidence of anal tone, or of anal-cutaneous and bulbo-cavenosous reflexes.

Laboratory tests, including blood count, liver and kidney functions were normal, with the exception of leucocytosis during the first days. Chest $\mathrm{X}$-rays showed patchy consolidations in the upper lobe of the right lung. Initial CSF examination taken on the first day in the hospital revealed $170 \mathrm{wbc} / \mathrm{cc}$ with $80 \%$ neutrophils and $20 \%$ monocytes, glucose $=71 \mathrm{mg} \%$, protein $=75 \mathrm{mg} \%$. Gram's stain was negative, and bacterial and fungal cultures were also negative. The second CSF examination carried out 2 days later demonstrated $218 \mathrm{wbc} / \mathrm{cc}$ with $88 \%$ monocytes and $12 \%$ monocytes, glucose $=79 \mathrm{mg} \%$, protein $=39 \mathrm{mg} \%$. CSF IgG was elevated, while oligoclonal bands were negative. Antigens to herpes simplex were $1 / 64$ in serum and $1 / 2$ in CSF, while IgG antigens for Q-fever were $1 / 100$ in serum (all values within normal limits). A series of serological tests for Weil-Felix, vidal, brucella, leptospira, legionella, psytacosis, chlamydia and trychomonas were negative on all accounts,

Table Sequential laboratory finding for mycoplasma infection.

\begin{tabular}{lcccc}
\hline $\begin{array}{l}\text { Hospital } \\
\text { day }\end{array}$ & $\begin{array}{c}\text { Cold agglutin } \\
\text { (serum) }\end{array}$ & $\begin{array}{c}\text { ELISA- } \\
\text { IgM to MP } \\
\text { sera }\end{array}$ & \multicolumn{2}{c}{ Compliment fixation } \\
serum MP & CSF MP \\
\hline 2 & --- & $-1++$ & $1 / 970$ & --- \\
4 & $1 / 64$ & ++ & -- & $1 / 920$ \\
6 & --- & --- & $1 / 480$ & $1 / 24$ \\
26 & $1 / 34$ & --- & --- & --- \\
\hline
\end{tabular}

as was an isotope examination with Galium scan (Table). Spinal X-rays and a CT scan of the dorsal and lumbar spine, and also a myelogram with Amipac were normal. Electromyography of the upper extremities showed no pathological findings, and the upper limb conduction velocities were normal. In the lower limbs however, peroneal F-wave responses were absent bilaterally. Needle EMG examination was within normal limits. An IVP showed no pathology. A urocystogram showed a hypotonic bladder, without signs of reflux or of trabaculations.

On the second day of hospitalisation the patient complained of numbness in the hands and the sensory deficit level rose to T3. There were no respiratory difficulties. A week later the sensory level had returned to T6 on the left and T8 on the right. The patient was treated with Doxylin (Doxycycline) and steroids. The consolidations in the right lung disappeared. Six months later the patient was confined to a wheelchair in a stable posture and was independent for self care; she had complete paraplegia; with a sensory level at T8, and was incontinent for both bladder and bowel functions. 
An MRI performed in the L-5-D-6 segment 3 months after admission revealed no pathological changes.

\section{Discussion}

ATM is a disorder of the spinal region of the CNS. Since the mortality is low, there are few reports of post-mortem examinations; these describe conditions ranging from normal to haemorrhagic and necrotising lesions (Tyler et al., 1986; Jain et al., 1983). Examinations on human patients have included clinical tests, CT scans, myelography and arteriography but without any pathological traits specific to the disease.

There have been two attempts to explain the pathogenesis of the ATM lesion. Firstly, it has been thought to be due to the presence of an infectious organism in the CNS or in adjacent blood vessels (Jain et al., 1983). Alternatively, the lesion in the spinal cord may be due to an immune system reaction. Abramsky (Abramsky and Teitelbaum, 1977) has stressed the importance of cell mediated auto-immune mechanisms. The immune system can be stimulated by penetration of the body by an infecting agent or by an auto-immune reaction of the body itself (Berman and Feldman, 1981; Rothstein and Kenny, 1979). This theory suggests that the presence of MP triggers the immune system to react, causing ATM to develop as an auto-immune reaction.

An MP infection can be diagnosed (Clyde, 1980; Azimi et al., 1984) by isolation of the organism from the infected area. There is only one report of the isolation of MP from the CSF (Fleischauer et al., 1972). The diagnosis can also be established by demonstrating a fourfold rise in specific antibody titres (Clyde, 1980; Nicholson, 1977). A single titre of 1:64 or higher of the specific antibody or cold agglutinin is suggestive of MP diagnosis. Antibody rise detected by any one of the various methods such as growth inhibition test, immunofluorescence, complement fixation $(\mathrm{CF})$, and gel diffusion tests without isolation of the organism are often used for identification of MP infection (Azimi et al., 1984). In the present case, diagnosis was made on the following grounds: pneumonia of the upper lobe of the right lung which reacted quickly to treatment with tetracyclines, strong reaction of cold agglutinin, complement fixation reaction inclusive of CSF and IGM specific to MP by ELISA techniques. (Table) It follows that the patient described here suffered from ATM preceded by an active MP infection.

In the present case, the clinical course showed ATM associated with MP and little recovery; the girl was left with a flaccid paraplegia, and thus differed from other cases in the literature which all reported complete or near complete recovery.

\section{References}

Abramsky O, Teitelbaum D 1977 The autoimmune features of acute transverse myelopathy. Annales of Neurology 2:36-40.

Azimi PH, Chase PA, Petru AM 1984 Mycoplasmas: their role in pediatric disease. Current Problems in Pediatrics 14 (8): 1-46.

Behan PO, Feldman RG, Segerra JM, Draper IT 1986 Neurological aspects of mycoplasmal infection. Acta Neurologica Scandinavica 74:314-322.

Berman M, Feldman S, Alter M, Zilber N, Kahana E 1981 Acute transverse myelitis: incidence and etiologic considerations. Neurology 31:966-971.

CLYDE WA 1980 Neurological syndromes and mycoplasmal infections (editorial). Archives of Neurology $37: 65-66$. 
Fleischauer P, Huber H, Martens H, Seithi KK 1972 Nachweis von Mycoplasma Pneumoniae in Liquor bei Akuter Polyneuritis. Deutsche Medizinische Wochenschrift 117:687-689.

JAIN AP, GUPTA OP, JAJOO UN 1983 A study of some prognostic factors in acute transverse myelitis. Fournal of the Association Physicians of India 31:497-499.

KLimeK JJ, RuSsman BS, QUINTILIANI R 1976 Mycoplasma pneumoniae meningoencephalitis and transverse myelitis in association with low cerebro-spinal fluid glucose. Pediatrics 58:133-135.

Lipton HL, TEASDALl RD 1973 Acute transverse myelopathy in adults. Archives of Neurology 28:252257.

MacFarlane PI, Miller V 1984 Transverse myelopathy associated with Mycoplasma pneumoniae infection. Archives of Disease in Childhood 59:80-82.

MERLI GJ, STAAS WE 1985 Acute transverse myelopathy association with body position. Archives of Physical Medicine and Rehabilitation 66:325-328.

Nagaswami S, KePes J, Foster DB, Twemlow SW 1973 Necrotizing myelitis: A clinico-pathologic report of two cases associated with Diplococcus pneumoniae and mycoplasma pneumoniae infections. Transactions of the American Neurological Society 48:290-292.

NichOLSON G 1977 Transverse myelitis complicating mycoplasma pneumoniae infection. Postgraduate Medical fournal 53:86-87.

RoTHSTEIN TL, KENNY GE 1979 Cranial neuropathy, myeloradiculopathy and myositis: complications of mycoplasma pneumoniae infection. Archives of Neurology 36:476-477.

TYLER KL, GROSS RA, CASCINO GD 1986 Unusual viral causes of transverse myelitis: Hepatitis A virus and cytomegalovirus. Neurology 36:855-858.

Westenfelder, GO, AKey T, CoRWIN SJ, Vick NA 1981 Acute transverse myelitis due to mycoplasma pneumoniae infection. Archives of Neurology 38:317-318. 